\title{
Rearrangements of $\mathrm{N}$-Acyl Isothioureas. Alternate Access to Acylguanidines from Cyanamides
}

\author{
Giovanni Maestri, ${ }^{\dagger}$ Marie-Hélène Larraufie, ${ }^{\dagger}$ Cyril Ollivier, ${ }^{\dagger}$ Max Malacria, ${ }^{\dagger}$ \\ Louis Fensterbank, ${ }^{\dagger}$ and Emmanuel Lacôte ${ }^{\star,+, \neq}$
}

UPMC Université Paris 06, IPCM (UMR 7201), 4 Pl. Jussieu, C. 229, 75005 Paris, France, and ICSN CNRS, Bât. 27, Avenue de la Terrasse, 91198 Gif sur Yvette Cedex, France

emmanuel.lacote@univ-lyon1.fr

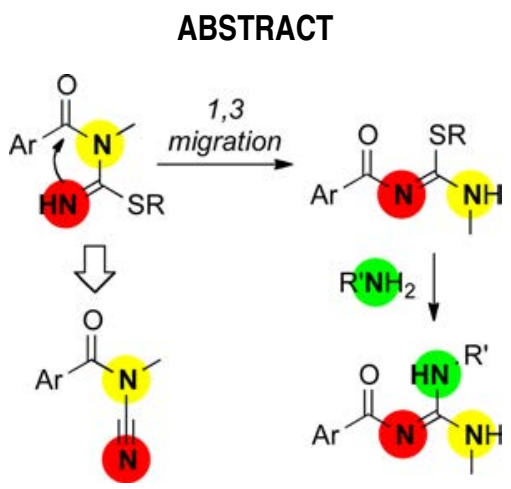

We report a tin-free one-pot radical approach to the synthesis of $\mathrm{N}$-acyl isothioureas and acylguanidines from $\mathrm{N}$-acyl cyanamides. Photoactivated reduction of aromatic disulfides in the presence of Hünig's base results in hydrothiolation of the cyanamide moiety, followed by spontaneous 1,3migration of the acyl group. Onward reaction of the isothioureas obtained with amines led to the corresponding $N$-acylguanidines, where the acyl group is attached to the nitrogen atom formerly at the cyano-end of the starting material.

Cyanamides are attractive $1 \mathrm{C}-2 \mathrm{~N}$ building blocks for nitrogen-rich molecules, whose reactivity can be further tuned by installing an acyl group next to the $\mathrm{sp}^{2}$ nitrogen. ${ }^{1}$ We have studied the reactivity of $\mathrm{N}$-acylcyanamides as partners for radical cascade reactions. This allowed us to selectively prepare quinazolinones ${ }^{2}$ as well as cyclic $N$-acyl guanidines. ${ }^{3}$

In the latter case, the acyl group in the acylguanidine formed was attached to the nitrogen that was bearing the cyanide moiety (Scheme 1a, highlighted in yellow). In the present communication we now report on the preparation of isomeric acylguanidines in which the acyl group is positioned

\footnotetext{
${ }^{\dagger}$ UPMC Université Paris 06.
}

ICSN CNRS.

(1) Larraufie, M.-H.; Maestri, G.; Malacria, M.; Ollivier, C.; Fensterbank, L.; Lacôte, E. Synthesis 2012, 44, 1279-1292.

(2) (a) Servais, A.; Azzouz, M.; Lopes, D.; Courillon, C.; Malacria, M. Angew. Chem., Int. Ed. 2007, 46, 576-579. (b) Larraufie, M.-H.; Courillon, C.; Ollivier, C.; Lacôte, E.; Malacria, M.; Fensterbank, L. J. Am. Chem. Soc. 2010, 132, 4381-4387. For an $\mathrm{SmI}_{2}$-mediated radical cascade involving cyanamides, see also (c) Hu, Z.; Li, S.-d.; Hong, P.-z. Arkivoc 2010, 171-177.

(3) Larraufie, M.-H.; Ollivier, C.; Fensterbank, L.; Malacria, M.; Lacôte, E. Angew. Chem., Int. Ed. 2010, 49, 2178-2181. on the nitrogen of the cyanide moiety (Scheme 1b, highlighted in red).

Our initial goal was to extend the scope of the radical cascades involving $N$-acyl cyanamides to thiyl radicals with the aim of accessing polycyclic isothioureas such as $\mathbf{A}$ (Table 1), for which no radical synthesis has been reported to date. Isothioureas are great ligands for transition metals, ${ }^{4}$ precursors of guanidines, ${ }^{5}$ and organocatalysts for asymmetric reactions. ${ }^{6}$

Reaction of substrate 1a with diphenyldisulfide was attempted under various conditions designed to generate the corresponding thiyl radical (Table 1). No conversion was observed when using AIBN, even in large excess

(4) Srinivas, K.; Srinivas, P.; Prathima, P. S.; Balaswamy, K.; Sridhar, B.; Rao, M. M. Catal. Sci. Technol. 2012, 2, 1180-1187.

(5) (a) Berlinck, R. G. S.; Kossuga, M. H.; Nascimento, A. M. Sci. Synth. 2005, 18, 1077-1116. (b) Manimala, J. C.; Anslyn, E. V. Eur. J. Org. Chem. 2002, 3909-3922.

(6) (a) Belmessieri, D.; Morrill, L. C.; Simal, C.; Slawin, A. M. Z.; Smith, A. D. J. Am. Chem. Soc. 2011, 133, 2714-2720. (b) Bumbu, V. D.; Birman, V. B. J. Am. Chem. Soc. 2011, 133, 13902-13905. (c) Taylor, J. E.; Bull, S. D.; Williams, J. M. W. Chem. Soc. Rev. 2012, 41, 2109-2121. 
Scheme 1. Access to $N$-Acylguanidines from $\mathrm{N}$-Acyl-Cyanamides
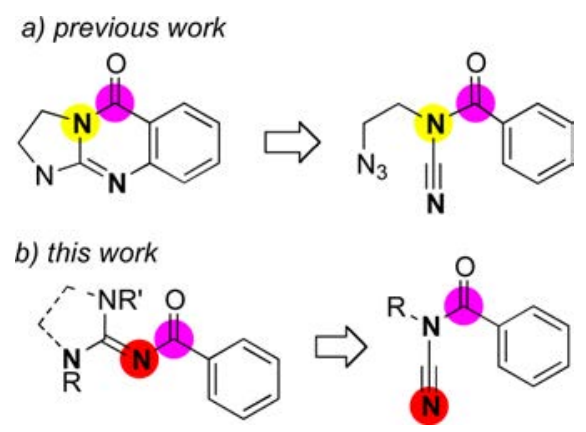

(entries 1-2). Introduction of tributyltin hydride was also unsuccessful, and pushing the conditions resulted only in some degradation of 1a (entries 3-5). Neither triethylborane $/ \mathrm{O}_{2}$ initiation nor irradiation (Xenon lamp) led to observable conversion (entries 6-8).

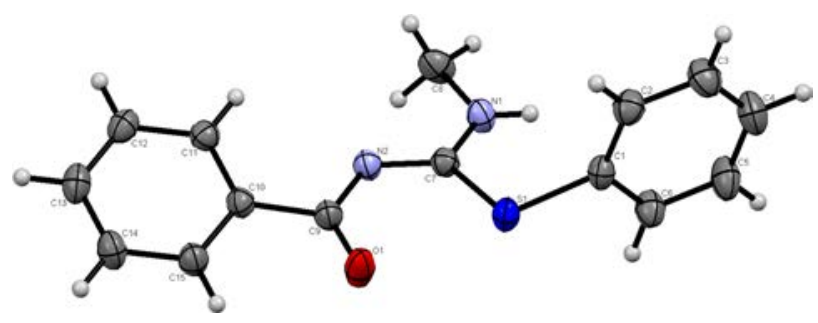

Figure 1. X-ray structure of 2.

We then turned to conditions introduced by Ishibashi for the formation of thiyl radicals via single electron transfer to the corresponding disulfides from an excess amine. ${ }^{7}$ Irradiation with a $14 \mathrm{~W}$ lightbulb of a triethylamine solution of 1a in the presence diphenyldisulfide delivered $\mathbf{2}$ in $29 \%$ yield (entry 9). When 1a was heated at reflux in tert-butanol in the presence of only 2 equiv of $\mathrm{NEt}_{3}$, almost complete conversion was observed, but only $21 \%$ of product was measured by NMR spectroscopy (entry 10).

We found that $N, N$-diisopropylethylamine (DIPEA) in $\mathrm{MeCN}$ at reflux was a better combination to generate $\mathbf{2}$ (36\%, entry 11). Further optimization showed that an excess of DIPEA and irradiation with a $14 \mathrm{~W}$ light bulb at rt yielded $78 \%$ of 2 (entries $12-13$ ). In particular, the mode of irradiation proved to be a key factor to obtain a synthetically useful yield (see below).

The NMR signals of the major product obtained (2) were too broad for unambiguous assignment. However, HRMS analysis indicated that the product had two hydrogen atoms more than was expected for $\mathbf{A}$. We eventually obtained crystals of $\mathbf{2}$ suitable for X-ray diffraction, and this allowed us to elucidate its structure (Figure 1).

(7) Taniguchi, T.; Fujii, T.; Idota, A.; Ishibashi, H. Org. Lett. 2009, 11, 3298-3301.
Table 1. Discovery and Optimization of the Migration of Hydrothiolated $N$-Acylcyanamides

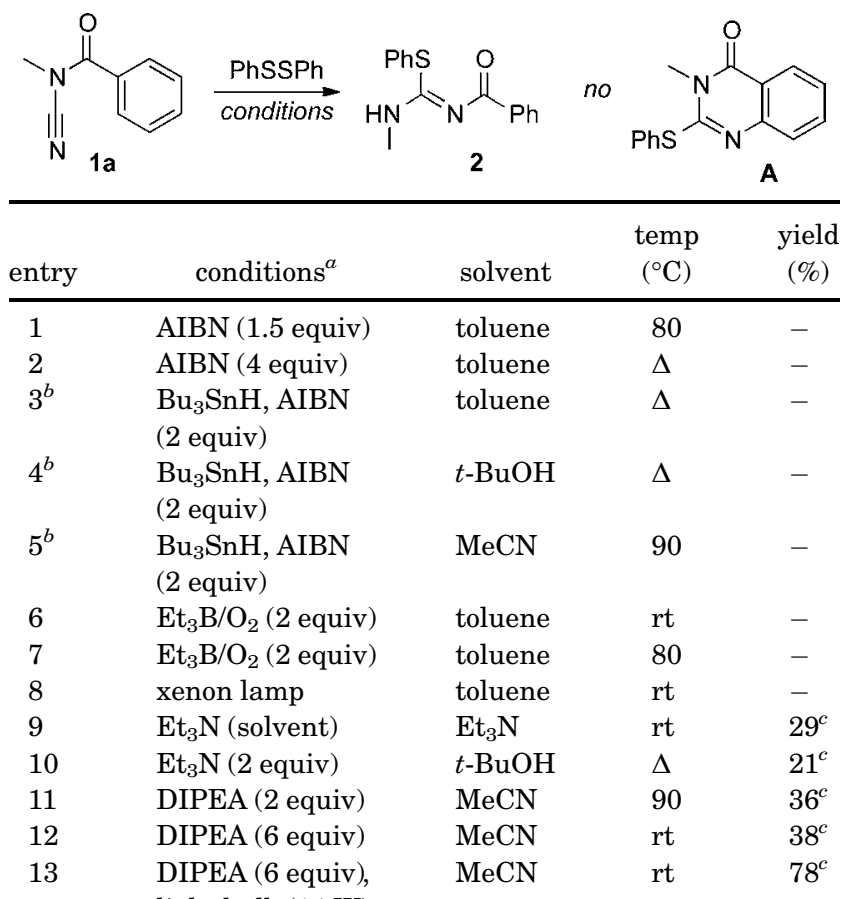

${ }^{a}$ Reactions conditions: 1a $(0.1 \mathrm{mmol}, 0.1 \mathrm{M}$ solution $), \mathrm{PhSSPh}$ ( 2 equiv), and reagents indicated in the table. Yield given for product 2. ${ }^{b}$ Slow addition $(0.05 \mathrm{mmol} / \mathrm{h}) .{ }^{c 1} \mathrm{H}$ NMR yield using $\mathrm{MeNO}_{2}$ as an internal standard.

Compound 2 turned out to be an acyclic $N$-acyl isothiourea in which the acyl moiety substituted the imino nitrogen atom, instead of the expected amino group, as would have been the case in the simple hydrothiolation of the starting cyanamides. This reactivity has not previously been reported.

The carbon-nitrogen bond lengths of the imino and the amino groups of 2 are very similar (1.31 and $1.33 \AA$ respectively). These distances are both slightly higher than usual carbon-nitrogen double bonds, as well as significantly shorter than a usual single bond (for example the $\mathrm{N}-\mathrm{C}$ (methyl) bond length is $1.47 \AA$ ), suggesting a tautomeric equilibrium. This is further supported by two distinct ${ }^{1} \mathrm{H}$ signals $\left(\delta 11\right.$ and $5.5 \mathrm{ppm}$ in $\mathrm{CDCl}_{3}$ ) for the labile proton, partially present on both nitrogens. Finally, the isothiourea is trans.

With the optimized reaction conditions in hand, we examined the scope of the reaction (Table 2). Different substituents can be installed on the cyanamide (on the nitrogen and/or the acyl group), as well as on the disulfide.

The reaction did not work with alkyldisulfides, so we concentrated on diversely substituted aryldisulfides. Electronrich aryl disulfides $\left(p-\mathrm{MeC}_{6} \mathrm{H}_{4}, p-\mathrm{MeOC}_{6} \mathrm{H}_{4}\right)$ led to good yields of rearranged adducts (entries 4-6, 8-9, 14). In contrast, the electron-deficient aryldisulfide $\left(p-\mathrm{Cl}-\mathrm{C}_{6} \mathrm{H}_{4} \mathrm{~S}\right)_{2}$ did not add well to cyanamide $1 \mathbf{a}(25 \%$, entry 2$)$.

At the acyl site, electron-rich arenes and heteroarenes delivered good yields of the expected adducts in a fast 
Table 2. Scope of the Rearranged $N$-Acyl Isothiourea Synthesis

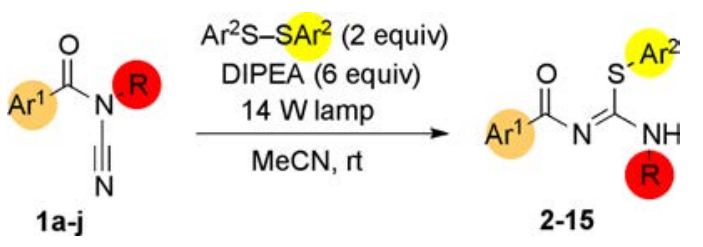

\begin{tabular}{|c|c|c|c|c|c|c|}
\hline entry & SM & $\mathrm{Ar}^{1}$ & $\mathrm{R}$ & $\mathrm{Ar}^{2}$ & prod. & $\begin{array}{l}\text { yield } \\
(\%)\end{array}$ \\
\hline 1 & $1 \mathbf{a}$ & $\mathrm{Ph}$ & $\mathrm{Me}$ & $\mathrm{Ph}$ & 2 & 78 \\
\hline 2 & $1 \mathrm{a}$ & $\mathrm{Ph}$ & $\mathrm{Me}$ & 4-Cl- $\mathrm{C}_{6} \mathrm{H}_{4}$ & 3 & 25 \\
\hline 3 & $1 b$ & $\mathrm{Ph}$ & $\mathrm{Bn}$ & $\mathrm{Ph}$ & 4 & 81 \\
\hline 4 & $1 b$ & $\mathrm{Ph}$ & $\mathrm{Bn}$ & $4-\mathrm{Me}-\mathrm{C}_{6} \mathrm{H}_{4}$ & 5 & 83 \\
\hline 5 & $1 c$ & $\mathrm{Ph}$ & Et & $4-\mathrm{Me}-\mathrm{C}_{6} \mathrm{H}_{4}$ & 6 & 82 \\
\hline 6 & $1 \mathrm{c}$ & $\mathrm{Ph}$ & Et & $\begin{array}{l}4-\mathrm{MeO}- \\
\mathrm{C}_{6} \mathrm{H}_{4}\end{array}$ & 7 & 79 \\
\hline 7 & $1 d$ & $\mathrm{Ph}$ & $c-\operatorname{Pr}$ & $\mathrm{Ph}$ & 8 & 38 \\
\hline 8 & $1 e$ & $\mathrm{Ph}$ & $\mathrm{Ph}$ & $4-\mathrm{Me}-\mathrm{C}_{6} \mathrm{H}_{4}$ & 9 & 68 \\
\hline 9 & $\mathbf{1 f}$ & 2 -furyl & $\mathrm{Et}$ & $\begin{array}{l}\text { 4-MeO- } \\
\mathrm{C}_{6} \mathrm{H}_{4}\end{array}$ & 10 & 92 \\
\hline 10 & $\mathbf{1 f}$ & 2 -furyl & Et & $\mathrm{Ph}$ & 11 & 93 \\
\hline 11 & $1 \mathrm{~g}$ & 2-thienyl & $\mathrm{Et}$ & $\mathrm{Ph}$ & 12 & 86 \\
\hline 12 & $1 \mathrm{~h}$ & 4-pyridyl & $\mathrm{Et}$ & $\mathrm{Ph}$ & 13 & 84 \\
\hline 13 & $\mathbf{1 i}$ & 3-pyridyl & $\mathrm{Et}$ & $\mathrm{Ph}$ & 14 & 60 \\
\hline 14 & $\mathbf{1 j}$ & $4-\mathrm{MeO}-\mathrm{C}_{6} \mathrm{H}_{4}$ & $\mathrm{Et}$ & $\begin{array}{l}\text { 4- } \mathrm{MeO}- \\
\mathrm{C}_{6} \mathrm{H}_{4}\end{array}$ & 15 & 86 \\
\hline
\end{tabular}

reaction (for example, see entries 9 and 14). With electronpoor arenes (4-nitrophenyl and 2,6-difluorophenyl), the reactions were sluggish, although pyridyl reagents worked satisfactorily (entries 12-13).

Also, various alkyl R substituents on the nitrogen of the reacting $N$-acylcyanamides are tolerated by the reaction. The reaction worked with aniline derivative 1e (entry 8), but the yield dropped sharply with cyclopropylamine substrate 1d (entry 7). We attributed the stereochemistry by analogy to that of $\mathbf{2}$. However, the NMR spectra of isothioureas are messy, certainly reflecting rotameric as well as tautomeric equilibria.

Isothioureas are the precursors of choice to guanidines. ${ }^{8}$ We thus wondered whether $N$-acylguanidines could be prepared from 2-15. In support of this possibility was the fact that the $\mathrm{C}-\mathrm{S}$ bond is slightly elongated $(1.79 \AA$, Figure 1).

The reaction of 2 with 1 equiv of benzylamine in the presence of triethylamine (1 equiv) and catalytic DMAP $(30 \mathrm{~mol} \%)$ at $\mathrm{rt}$ readily afforded guanidine $\mathbf{1 6 a}$ in $77 \%$ yield (together with $18 \%$ of benzylbenzamide, likely coming from transamidation at the carbonyl group of 2).

Gratifyingly, an optimized one-pot procedure for the direct synthesis of the $\mathrm{N}$-acylguanidines from the starting cyanamides was devised. In a typical experiment, after completion of the sulfenylation, benzylamine and DMAP

(8) For a recent review on biological properties of guanidines: (a) Castagnolo, D.; Schenone, S.; Botta, M. Chem. Rev. 2011, 111, 5247-5300. (b) Ishikawa, T. Chem. Pharm. Bull. 2010, 58, 1565-1568.
(10 mol \%) were added to the reaction mixture to afford $16 a$ in $73 \%$ yield.

The reaction was generally efficient, except when an electron-poor disulfide was used (Table 3 , entry 2 ) and in the case of cyclopropylamine cyanamide 1d (entry 8). The low yield likely reflects the inefficiency of the initial thiol addition.

Table 3. One-Pot Synthesis of Acylguanidine from $N$-Acylcyanamides
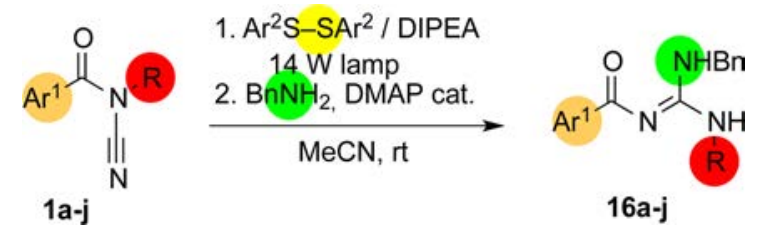

\begin{tabular}{lclcc}
\hline entry & substrate $^{a}$ & \multicolumn{1}{c}{$\mathrm{Ar}^{2}$} & product & yield (\%) \\
\hline 1 & $\mathbf{1 a}$ & $\mathrm{Ph}$ & $\mathbf{1 6 a}$ & 73 \\
2 & $\mathbf{1 a}$ & $4-\mathrm{Cl}-\mathrm{C}_{6} \mathrm{H}_{4}$ & $\mathbf{1 6 a}$ & 19 \\
3 & $\mathbf{1 b}$ & $\mathrm{Ph}$ & $\mathbf{1 6 b}$ & 69 \\
4 & $\mathbf{1 b}$ & $4-\mathrm{Me}-\mathrm{C}_{6} \mathrm{H}_{4}$ & $\mathbf{1 6 b}$ & 70 \\
5 & $\mathbf{1 c}$ & $\mathrm{Ph}$ & $\mathbf{1 6 c}$ & 65 \\
6 & $\mathbf{1 c}$ & $4-\mathrm{Me}-\mathrm{C}_{6} \mathrm{H}_{4}$ & $\mathbf{1 6 c}$ & 65 \\
7 & $\mathbf{1 c}$ & $4-\mathrm{MeO}-\mathrm{C}_{6} \mathrm{H}_{4}$ & $\mathbf{1 6 c}$ & 61 \\
8 & $\mathbf{1 d}$ & $\mathrm{Ph}$ & $\mathbf{1 6 d}$ & 29 \\
9 & $\mathbf{1 f}$ & $\mathrm{Ph}$ & $\mathbf{1 6 f}$ & 67 \\
10 & $\mathbf{1 f}$ & $4-\mathrm{MeO}-\mathrm{C}_{6} \mathrm{H}_{4}$ & $\mathbf{1 6 f}$ & 65 \\
11 & $\mathbf{1 g}$ & $\mathrm{Ph}$ & $\mathbf{1 6 g}$ & 37 \\
12 & $\mathbf{1 h}$ & $\mathrm{Ph}$ & $\mathbf{1 6 h}$ & 73 \\
13 & $\mathbf{1 i}$ & $\mathrm{Ph}$ & $\mathbf{1 6 i}$ & 52 \\
14 & $\mathbf{1 j}$ & $\mathrm{Ph}$ & $\mathbf{1 6 j}$ & 66 \\
& & &
\end{tabular}

Employing diamines, it was possible to obtain the bisacylguanidines 17-19 (Scheme 2). Because the latter have the same rigid N,O scaffold as many ligands of the bimetallic and/or bischelated complexes used for catalytic epoxidation or insertion polymerization, ${ }^{9}$ our method could provide an entry to such complexes.

One can also take advantage of the nucleophilicity of the acylguanidine to obtain cyclic adducts (Scheme 3). Employing bromopropylamine positions a leaving group on the adduct, which can cyclize to $\mathbf{2 0}$ in $70 \%$ overall yield (Scheme 3a). Similarly, aza-imidazolones such as $\mathbf{2 1}$ can be obtained from C-protected amino acids after formation of a $\operatorname{lactam}(57 \%$ for the phenylalanine derivative, Scheme $3 \mathrm{~b})$. Interestingly, C-protected lysine is a diamine which led to 22 , where the two amino groups reacted differently. The side-chain amino led to a regular acylguanidine, and the amino acid led to an aza-imidazolone. An X-ray structure was obtained for 21 (see Supporting Information). The

(9) For reviews: (a) Trifonov, A. A. Coord. Chem. Rev. 2010, 254, 1327-1347. (b) Gupta, K. C.; Sutar, A. K. Coord. Chem. Rev. 2008, 252, 1420-1450. Recent examples with bis-chelating ketoiminates: (c) Kirillov, E.; Roisnel, T.; Carpentier, J.-F. Organometallics 2012 , 31, 3228-3240. (d) Song, D.-P.; Shi, X.-C.; Wang, Y.-X.; Yang, J.-X.; Li, Y.-S. Organometallics 2012, 31, 966-975. 
Scheme 2. Access to Bis $N$-Acylguanidines

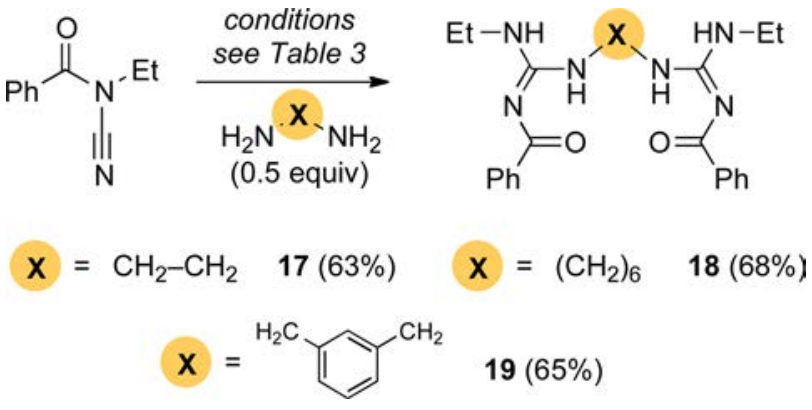

Scheme 3. Access to Functionalized $N$-Acylguanidines through Tandem Reactions Involving Subtitution (a) or Transamidation (b)

a)

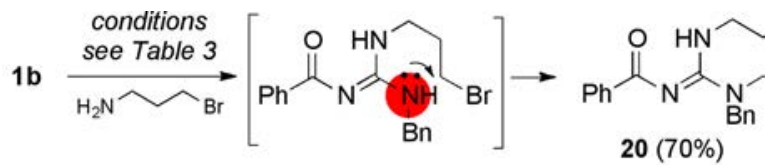

b)

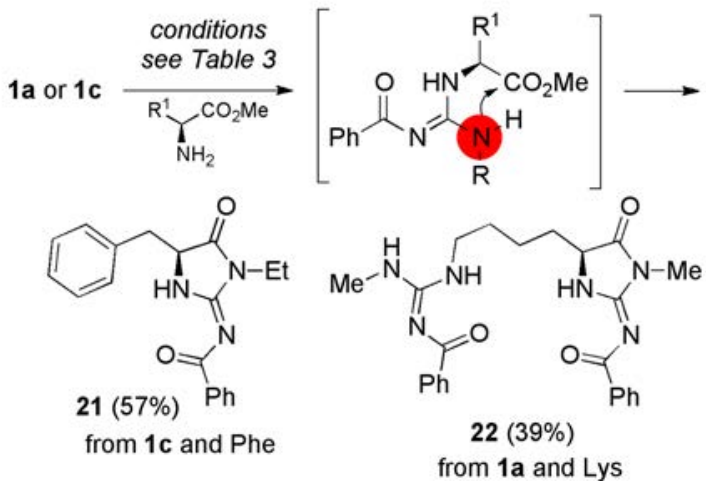

configurations of the other guanidines were attributed by analogy with 21.

A tentative mechanism for the rearrangement of acylcyanamides is presented in Scheme 4.

We believe the reaction starts with single electron transfer from DIPEA to the disulfide, as suggested by Ishibashi. ${ }^{7}$ This would generate a radical anion that would collapse to both a thiyl radical and a thiolate. This electron transfer would not be possible for the less readily reduced

(10) Christensen, T. B.; Daasbjerg, K. Acta Chim. Scand. 1997, 51, 307-317.

(11) Analogous electron transfer with even less readily reduced copper: Sreenath, K.; Suneesh, S. C.; Ratheesh Kumar, V. K.; Gopidas, K. R. J. Org. Chem. 2008, 73, 3245-3251.
Scheme 4. Mechanistic Proposal

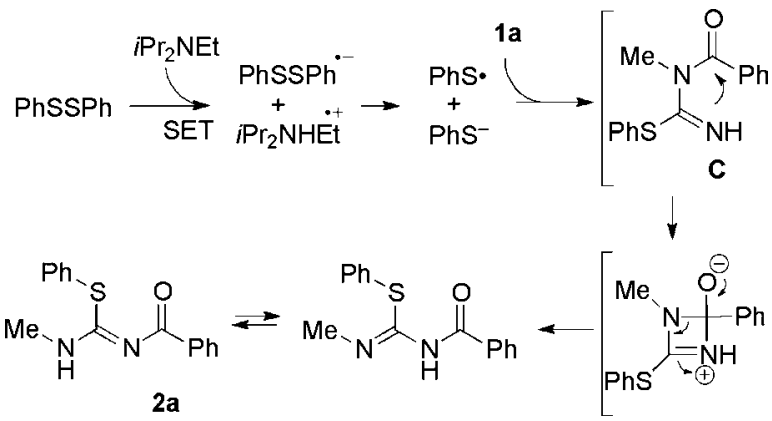

dialkyldisulfides, ${ }^{10}$ which did not add to the cyanamides under the reported conditions. No conversion was observed in the presence of stoichiometric soluble $\mathrm{Ag}(\mathrm{I})$ salts, which are more easily reduced than diphenyldisulfide, ${ }^{11}$ or TEMPO. Both additives would capture the electron instead of the diaryldisulfides.

In contrast to our previous free-radical cascades, ${ }^{1-3}$ this appears to trigger hydrothiolation of the acylcyanamide to C, without radical dearomatization. The exact mechanism for that step is still not clear, but after that, $\mathbf{C}$ undergoes spontaneous migration, likely by nucleophilic attack of the imine on the carbonyl, followed by fragmentation to $\mathbf{2 a}$. The absence of cyclopropyl ring-opened products (see $\mathbf{2 d}$ ) makes it unlikely that the rearrangement proceeds via a radical pathway.

In conclusion we have developed a new method for the synthesis of $N$-acyl isothioureas and $N$-acyl cyanamides from $N$-acylcyanamides with geometries different from those accessible through our previous free radical cyclizations. This opens access to a large family of compounds with structures potentially of interest as organocatalysts or ligands for transition metals.

Acknowledgment. We thank CNRS, UPMC, IUF (M.M., L.F.), the région Ile-de-France (M.-H.L.), and ANR (Grant Credox) for funding of this work. Analytical equipment was generously offered through FR 2769. We thank Geoffroy Gontard (UPMC) for the X-ray diffraction analysis.

Supporting Information Available. Experimental procedure, characterization data, and NMR spectra for all new compounds. This material is available free of charge via the Internet at http://pubs.acs.org.

The authors declare no competing financial interest. 